\title{
Speckle-based three-dimensional velocity measurement using spatial filtering velocimetry
}

Iversen, Theis Faber Quist; Jakobsen, Michael Linde; Hanson, Steen Grüner

Published in:

Applied Optics

Link to article, DOI:

10.1364/AO.50.001523

Publication date:

2011

Document Version

Publisher's PDF, also known as Version of record

Link back to DTU Orbit

Citation (APA):

Iversen, T. F. Q., Jakobsen, M. L., \& Hanson, S. G. (2011). Speckle-based three-dimensional velocity measurement using spatial filtering velocimetry. Applied Optics, 50(11), 1523-1533.

https://doi.org/10.1364/AO.50.001523

\section{General rights}

Copyright and moral rights for the publications made accessible in the public portal are retained by the authors and/or other copyright owners and it is a condition of accessing publications that users recognise and abide by the legal requirements associated with these rights.

- Users may download and print one copy of any publication from the public portal for the purpose of private study or research.

- You may not further distribute the material or use it for any profit-making activity or commercial gain

- You may freely distribute the URL identifying the publication in the public portal

If you believe that this document breaches copyright please contact us providing details, and we will remove access to the work immediately and investigate your claim. 


\title{
Speckle-based three-dimensional velocity measurement using spatial filtering velocimetry
}

\author{
Theis F. Q. Iversen, ${ }^{1, \star}$ Michael L. Jakobsen, ${ }^{2}$ and Steen G. Hanson ${ }^{2}$ \\ 'OPDI Technologies A/S, Frederiksborgvej 399, 4000 Roskilde, Denmark \\ ${ }^{2}$ DTU Fotonik, Department of Photonics Engineering, Technical University of Denmark, \\ P.O. Box 49, DK-4000, Roskilde, Denmark \\ ${ }^{*}$ Corresponding author: ti@opdi-technologies.com
}

Received 5 November 2010; accepted 10 January 2011; posted 24 January 2011 (Doc. ID 137798); published 1 April 2011

\begin{abstract}
We present an optical method for measuring the real-time three-dimensional (3D) translational velocity of a diffusely scattering rigid object observed through an imaging system. The method is based on a combination of the motion of random speckle patterns and regular fringe patterns. The speckle pattern is formed in the observation plane of the imaging system due to reflection from an area of the object illuminated by a coherent light source. The speckle pattern translates in response to in-plane translation of the object, and the presence of an angular offset reference wave coinciding with the speckle pattern in the observation plane gives rise to interference, resulting in a fringe pattern that translates in response to the out-of-plane translation of the object. Numerical calculations are performed to evaluate the dynamic properties of the intensity distribution and the response of realistic spatial filters designed to measure the three components of the object's translational velocity. Furthermore, experimental data are presented that demonstrate full 3D velocity measurement. (C) 2011 Optical Society of America
\end{abstract}

OCIS codes: $\quad 030.1640,120.7250,030.1670,030.6140,030.6600$.

\section{Introduction}

The application of laser speckle patterns to measure in-plane motion of diffuse objects is well established in the literature and includes a multitude of methods. Methods such as speckle photography (SP) and electronic SP (ESP) [1] have been applied successfully to measure in-plane displacement fields of an imaged object under load. The related approach of speckle interferometry (SPI/ESPI) combines the speckle pattern with a reference wave incident on the image plane under an angle in order to measure the out-of-plane displacement by fringe analysis [2]. Furthermore, these two techniques have been combined partly to enhance the fringe analysis in applications where large in-plane displacements are

0003-6935/11/111523-11\$15.00/0

(C) 2011 Optical Society of America considered and partly to measure a full threedimensional (3D) displacement field [3]. However, these techniques are full-field measurements acquired by a digital camera or conventional photography. Therefore, they are limited by the fringe analysis to measure out-of-plane displacements on the order of a few wavelengths and are particularly not well suited for real-time studies and velocimetry.

Recently [4], it has been demonstrated that speckle patterns propagated in free space in combination with a conventional in-line Michelson interferometer realize single-point measurement of $3 \mathrm{D}$ displacement. However, this method will not be able to distinguish between translation and rotation of the object.

Another method that is dedicated to measure outof-plane velocity is coherent Doppler lidar [5,6]. Here, the out-of-plane displacement is limited $\bar{b} y$ the focal depth of the imaging system (typically on 
the order of a meter), and particle velocities on the order of several meters per second are measured in real time. Work has been carried out to measure small in-plane velocity components $[7,8]$ in these types of systems. However, the implementations are inherently single mode in the spatial domain, and all spatial information in the image plane is discarded. The same type of system can be modified to include the dynamic speckle field in the image plane and, under certain circumstances, can be used to measure in-plane motion [9].

We investigate, by means of numerical calculations and experiments, the use of an imaging system to form an image of an area of a diffusely scattering rigid object illuminated by a coherent light source. The speckle pattern that arises in the image plane is combined with a coherent reference wave that coincides with the speckle image under an angle. The resulting intensity distribution will exhibit two distinct features: (i) a speckle pattern that translates according to the in-plane motion of the object and (ii) a regular fringe pattern that arises due to the interference between the reference wave and the speckle pattern. The fringe pattern will translate in response to an out-of-plane motion of the object. Methods from spatial filtering velocimetry [10] are implemented to measure all three velocity components. A numerical model, based on the complex ABCD matrix formalism [11], is developed to investigate the frequency characteristics of the output signals of realistic spatial filters and used to identify a suitable experimental configuration. The in-plane motion is addressed experimentally by a CMOS camera where the spatial filtering is implemented in the postprocessing. Previously, such methods have been demonstrated $[10,12]$ and more recently described in a similar application [13]. The measurement of the out-of-plane motion is facilitated by an integrated optical spatial filter due to its high sensitivity [14]. The proposed method is intended for use in real-time studies where 3D translational velocity is of interest. Possible applications include remote sensing of structural dynamics, conditional monitoring, and fluid dynamics including aerosol flow. In the following section, the underlying theory of the numerical model is reviewed. In Section 3 the numerical model is presented and the results are discussed. The configuration of the experimental setup is based on the observations obtained from the numerical simulations and is described in Section 4. The experimental results are presented in Section 5 . A general discussion and concluding remarks are included in Section 6 .

\section{Theoretical Review}

In order to evaluate the performance of specific spatial filtering velocimetry methods that will be used to measure the velocity components of the object translation, a simple numerical model is developed based on the ABCD formalism. In Fig. 1 the geometry of the model is illustrated.

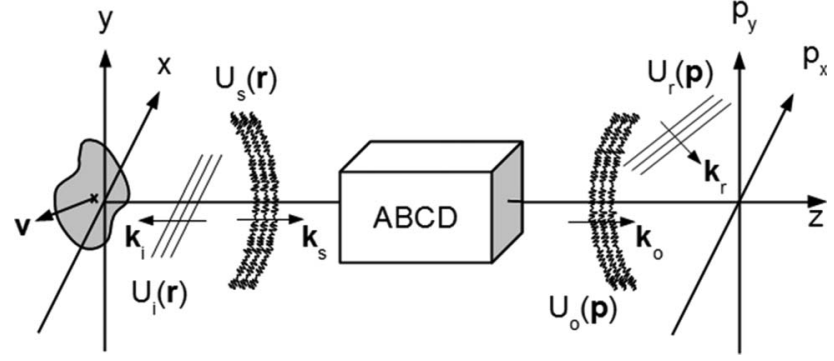

Fig. 1. ABCD model geometry.

A diffusing rigid object is located in the $(x, y)$ plane, translating with constant velocity $\mathbf{v}$ and illuminated by a field $U_{i}(\mathbf{r})$ of the form

$$
U_{i}(\mathbf{r})=E_{i} \exp \left(-\frac{\mathbf{r}^{2}}{w_{i}^{2}}\right),
$$

where $w_{i}$ is the $1 / e^{2}$ intensity radius. The scattered field $U_{s}(\mathbf{r}, t)$ can then be written as

$$
U_{s}(\mathbf{r}, t)=U_{i}(\mathbf{r}) \Psi(\mathbf{r}, t),
$$

where $\Psi(\mathbf{r}, t)$ is the complex reflection coefficient of the object. It is assumed that $|\Psi(\mathbf{r}, t)|=$ const. Furthermore, it is assumed that the object surface structure does not evolve in time but moves as a "frozen" pattern. This attribute can be expressed by $\Psi(\mathbf{r}, t+\tau)=\Psi(\mathbf{r}-\mathbf{v} \tau, t)$. The scattered field is propagated to the observation plane $\left(p_{x}, p_{y}\right)$ by means of the paraxial Green's function defined by an ABCD matrix representing an imaging system. Thus, the field in the observation plane at time $t$ can be obtained from

$$
U_{o}(\mathbf{p}, t)=\int_{-\infty}^{+\infty} \mathrm{d}^{2} \mathbf{r} U_{s}(\mathbf{r}) G(\mathbf{r}, \mathbf{p}),
$$

where

$G(\mathbf{r}, \mathbf{p})=-\frac{i k}{2 \pi B} \exp \left[-i k L^{\prime}\right] \exp \left[-\frac{i k}{2 B}\left(A \mathbf{r}^{2}-2 \mathbf{r} \cdot \mathbf{p}+D \mathbf{p}^{2}\right)\right]$.

The entities $A, B$, and $D$ represent the complex matrix elements of the optical system. The parameter $k$ is the optical wavenumber, and $L^{\prime}$ is the optical path length from the object to the observation plane. Additionally, the refractive indices in the object and observation plane have been assumed to be identical. In the following, a specific ABCD system is considered with the following elements:

$$
\begin{gathered}
A=-\frac{f_{2}}{f_{1}}, \\
B=\frac{-2 i f_{1} f_{2}}{k \sigma^{2}}, \\
D=-\frac{f_{1}}{f_{2}} .
\end{gathered}
$$


The ABCD system defined by Eqs. (5)-(7) constitutes a clean imaging system (i.e., a $4-\bar{f}$ system) with magnification $M=-f_{2} / f_{1}$ and a Gaussian-apodized aperture of $\sigma$ (1/e field radius) in the exact Fourier plane. Combining Eq. (4) with Eqs. (5)-(7), the Green's function for the imaging system becomes

$$
G(\mathbf{r}, \mathbf{p})=\frac{k^{2} \sigma^{2}}{4 \pi f_{1} f_{2}} \exp \left[-i k L^{\prime}\right] \exp \left[-\frac{k^{2} \sigma^{2}}{4 f_{2}^{2}}\left(\frac{f_{2}}{f_{1}} \mathbf{r}+\mathbf{p}\right)^{2}\right] .
$$

The field $U_{o}(\mathbf{p})$ is combined with a mutually coherent reference wave $U_{r}(\mathbf{p})$ at the observation plane. The reference wave is written as

$$
U_{r}(\mathbf{p})=E_{r} \exp \left(-\frac{\mathbf{p}^{2}}{w_{r}^{2}}-i k \boldsymbol{\theta} \cdot \mathbf{p}\right),
$$

where $w_{r}$ is the $1 / e^{2}$ intensity radius and $\theta=\left(\theta_{x}, \theta_{y}\right)$ is the vector angle of incidence of the reference wave relative to the optical axis (the $z$ axis) of the imaging system. Then the time-dependent intensity distribution in the observation plane can be obtained by

$$
I(\mathbf{p}, t)=\left|U_{o}(\mathbf{p}, t)+U_{r}(\mathbf{p})\right|^{2} .
$$

The power contained in each of the two contributions $U_{o}(\mathbf{p}, t)$ and $U_{r}(\mathbf{p})$ is defined in the following way:

$$
\begin{gathered}
P_{o}=\int_{-\infty}^{\infty} \mathrm{d}^{2} \mathbf{p}\left|U_{o}(\mathbf{p}, t)\right|^{2} . \\
P_{r}=\int_{-\infty}^{\infty} \mathrm{d}^{2} \mathbf{p}\left|U_{r}(\mathbf{p})\right|^{2} .
\end{gathered}
$$

The intensity distribution at a later time $t+\tau$ can be obtained by letting

$$
\Psi(x, y, t) \rightarrow \Psi\left(x-v_{x} \tau, y-v_{y} \tau, t+\tau\right) \exp \left(-i k v_{z} \tau\right),
$$

where it is assumed that $\mathbf{v}=\left(v_{x}, v_{y}, v_{z}\right)$ is constant. It is noted that the optical distance $L^{\prime}$ in Eq. (8) must be modified by $L^{\prime} \rightarrow L^{\prime}+v_{z} \tau$. The range of axial displacements that is investigated in this context will be limited, so that the total axial displacement $|\Delta z| \ll d_{o}$ where $d_{o}=4 f_{1}^{2} /\left(k \sigma^{2}\right)$ is the depth of focus of the imaging system. Conversely, it can be argued that the depth of focus effectively localizes the measurement volume, facilitating a pointlike measurement, which is an advantage in many applications. If axial displacement significantly exceeds $d_{o}$, the ABCD system described by Eqs. (5)_(7) must undergo modification to accommodate this. Such considerations are beyond the scope of this work.

Having obtained the dynamic intensity distribution $I(\mathbf{p}, t)$, determination of the temporal response of a spatial filter realized by a detector arrangement placed directly in the observation plane is called for.
As the intensity distribution is sheared across the spatial filter, the time-dependent photocurrent signal can be obtained from [10], except for a proportionality factor

$$
i\left(\mathbf{p}_{\mathbf{1}}\right)=\int_{-\infty}^{+\infty} \mathrm{d}^{2} \mathbf{p} I\left(\mathbf{p}_{\mathbf{1}}-\mathbf{p}\right) h(\mathbf{p}),
$$

where $\mathbf{p}_{\mathbf{1}}=M v t+\mathbf{p}_{\mathbf{0}}$ and $\mathbf{p}_{\mathbf{0}}$ is a constant vector.

The quantity $h(\mathbf{p})$ is the spatial filter response function. According to the convolution theorem, the power spectrum of the output signal described by Eq. (14) can be written as

$$
P(\boldsymbol{\xi})=\left|S_{I}(\boldsymbol{\xi})\right|^{2}|H(\boldsymbol{\xi})|^{2},
$$

where $\xi=\left(\xi_{x}, \xi_{y}\right)$ denotes the spatial frequency vector in the $\mathbf{p}$ plane and $\left|S_{I}(\boldsymbol{\xi}, t)\right|^{2}$ and $|H(\xi)|^{2}$ are the power spectral density of the intensity distribution and the spatial filter response function (the transfer function), respectively. The properties and configuration of the response function $h(\mathbf{p})$ can vary depending on the application, but in general it will consist of detector elements arranged in a periodic structure combining alternating elements with sum or differential amplifiers. The purpose of such an arrangement is to realize a narrow spatial bandpass filter that monitors the temporal phase evolution of the selected range of spatial frequencies. Accordingly, the detected photocurrent, i.e., the output signal of the spatial filter, will oscillate at a frequency proportional to the velocity component in the direction of the spatial filter periodicity. The frequency of this oscillation is given by the relation

$$
F=\frac{M v}{\Lambda},
$$

where $\Lambda$ is the spatial period of the spatial filter, $M$ is the magnification of the imaging system, and $v$ is the velocity component of the object in the direction of the periodicity of the spatial filter.

The detector arrangement that will be used here has been demonstrated by Schnell et al. [12]. The detector aperture function can be written as

$$
\begin{aligned}
h(\mathbf{p}) & =\left[h_{\mathrm{win}}(\mathbf{p}) \sum_{m=-\infty}^{\infty} \delta\left(p_{x}-m \Lambda_{x}\right)\right] \\
& \otimes\left[h_{\mathrm{unit}}\left(p_{x}\right) \otimes h_{\mathrm{det}}\left(p_{x}\right)\right],
\end{aligned}
$$

where

$$
\begin{gathered}
h_{\text {win }}(\mathbf{p})=\operatorname{Rect}\left(\frac{p_{x}}{L_{x}}\right) \operatorname{Rect}\left(\frac{p_{y}}{L_{y}}\right), \\
h_{\text {unit }}\left(p_{x}\right)=\delta\left(p_{x}+p_{\varphi}\right)-\delta\left(p_{x}-p_{\varphi}\right), \\
h_{\text {det }}\left(p_{x}\right)=\operatorname{Rect}\left(\frac{p_{x}}{W_{d}}\right),
\end{gathered}
$$

and $\delta(x)$ denotes the Dirac-delta function. Selecting $W_{d}=\Lambda_{x} / 4, p_{\varphi}=\Lambda_{x} / 4$ and $L_{x} \rightarrow N \Lambda_{x}$, the power spectral density of the response function will be given by 


$$
\begin{aligned}
|H(\xi)|^{2}= & \operatorname{sinc}^{2}\left(L_{y} \xi_{y}\right) \sum_{m=-\infty}^{\infty} \operatorname{sinc}^{2}\left[N\left(\Lambda_{x} \xi_{x}-m\right)\right][1 \\
& \left.-\cos \left(\pi \Lambda_{x} \xi_{x}\right)\right] \operatorname{sinc}^{2}\left(\frac{\Lambda_{x} \xi_{x}}{4}\right)
\end{aligned}
$$

where an unimportant proportionality factor has been omitted. The parameters $W_{d}$ and $p_{\varphi}$ are selected based on the experimental configuration used (see Section 4). A second detector arrangement is now interlaced with the first one, providing a second signal that will be in phase quadrature with the signal obtained from the first detector arrangement, facilitating an unambiguous directional discrimination of the translation. The two response functions of the detector arrangements are defined as $h_{0}(\mathbf{p}) \equiv h(\mathbf{p})$ and $h_{90}(\mathbf{p}) \equiv h\left(p_{x}-\Lambda_{x} / 4, p_{y}\right)$. The power spectral density will be identical for both filters, as only a complex phase factor distinguishes the spectrum of one from the other. The orientation of the detector arrangements defines the direction of the velocity components to be measured. Generally, the in-plane motion is measured with two orthogonally orientated spatial filters, placed directly in the observation plane and tuned to the speckle size. However, a spatial filter can attain any angle in the observation plane. This feature will be necessary for determining all three velocity components and can be accommodated by introducing a new coordinate system that incorporates an arbitrary rotation of the filter orientation, here implemented in the spatial frequency domain

$$
\begin{aligned}
& \xi_{x}^{\prime}=\xi_{x} \cos (\Omega)+\xi_{y} \sin (\Omega), \\
& \xi_{y}^{\prime}=\xi_{y} \cos (\Omega)-\xi_{x} \sin (\Omega) .
\end{aligned}
$$

Figure 2 shows the detector arrangement and orientation. In Fig. 3 , the power spectral density of an eight unit cell spatial filter is shown. The filter not only contains a spatial bandpass region around the first harmonic (fundamental) but also several higher harmonics of odd numbers are seen to contribute significantly. This is a well-known property of rectangular detector arrangements. It has been shown [14] that the $K$ th harmonics can, under certain conditions, be suppressed by selecting $W_{d}=\Lambda / K$. Given the detector configuration described above, the fourth harmonic will be suppressed but the third and fifth harmonics may constitute significant passbands in the spatial power spectrum of the spatial filter.

\section{Numerical Results}

To investigate the dynamic behavior of the intensity distribution in response to a $3 \mathrm{D}$ object translation and determine the temporal response of various spatial filtering implementations, a numerical model was developed. The object plane and the observation plane are both represented as matrices of $128 \times 128$ points. The spatial sampling periods are defined as $\Delta x$ and $\Delta y$ and correspond to the physical separation between sampling points (pixels) along the hori-

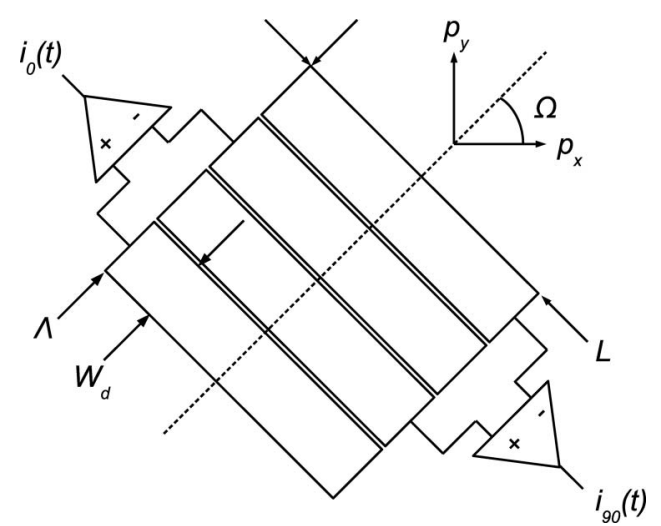

Fig. 2. Spatial filter unit cell combining both the $h_{0}(\mathbf{p})$ and $h_{90}(\mathbf{p})$ filters. The rotation angle of the detector arrangement in relation to the observation plane coordinate system is $\Omega$.

zontal and vertical directions, respectively. In this model, we select $\Delta x=\Delta y=5 \mu \mathrm{m}$.

The scattering properties of the object are defined by a random spatial distribution of scattering points in the object plane, having an identical scattering cross section. An individual scattering point (or particle) is represented by a single pixel in the numerical model. Each scattering point is attributed with a spatially delta-correlated complex random phase factor $\exp (-i \phi)$ where the phase $\phi$ is a stochastic variable described by a uniform probability distribution function (PDF) of the form

$$
\operatorname{PDF}(\phi)= \begin{cases}\frac{1}{2 \pi}, & -\pi \leq \phi \leq \pi \\ 0, & |\phi|>\pi\end{cases}
$$

When the imaging system is not able to resolve the individual scattering particle and the number of scattering particles is large, the resulting field distribution obtained in the observation plane will exhibit

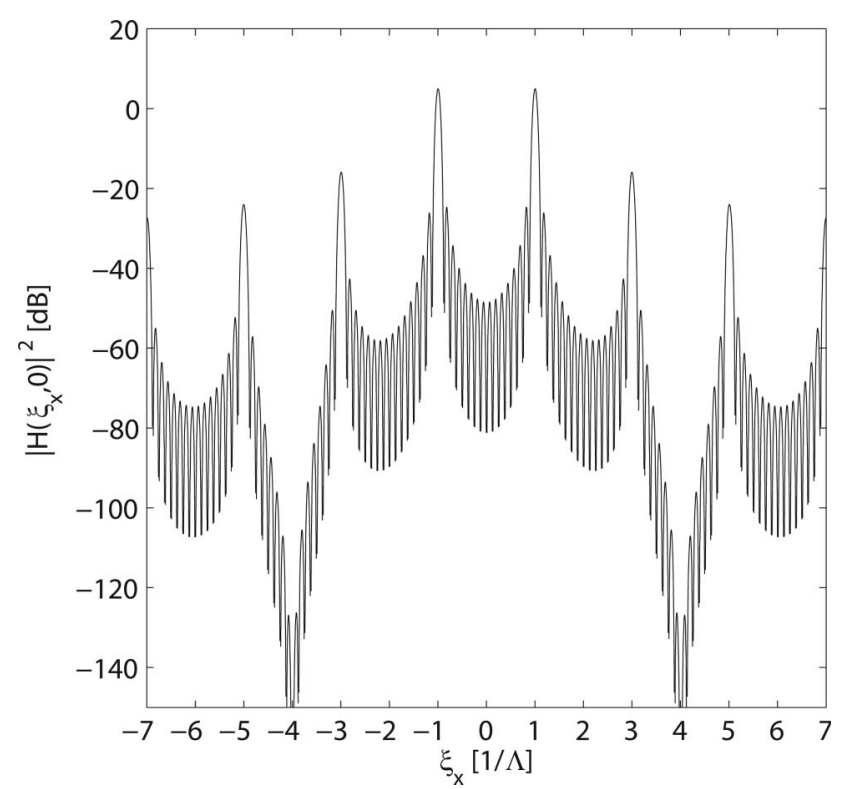

Fig. 3. Spatial power spectral function of the spatial filter described by Eq. (21) for $\Lambda \rightarrow \Lambda_{x}, L \rightarrow L_{y}, \Omega \rightarrow 0^{\circ}$, and $N=8$. 
circular complex Gaussian statistics, giving rise to a fully developed speckle pattern [15]. It is straightforward to calculate the intensity distributions at different times in the object plane as the field of scattering points is translated according to Eq. (13) combining Eq. (1)-(10). The model implemented here assumes that effects of shot noise and thermal noise are negligible. Furthermore, it must be noted that due to the now discrete nature of the object and observation planes, the integrals are replaced with a corresponding summation operation. For simplicity, $w=$ $w_{r}=\left(f_{2} / f_{1}\right) w_{o}, f_{1}=f_{2}=f$, and the aperture is apodized as a Gaussian function in the following. In Fig. 4 a representative simulated intensity distribution is shown. In the following, the parameters listed in Fig. 4 will be maintained.

It can be observed that the intensity distributions generally contain a random speckle pattern and a regular fringe pattern that is enclosed by the individual speckles. Furthermore, the entire speckle field is weighted by the image of the illumination spot on the object. The reference wave responsible for the regular fringe pattern formation exhibits a completely deterministic optical phase front across the observation plane, whereas the optical field distribution producing the speckle pattern exhibits an uncorrelated phase relationship between individual speckles. In the spatial frequency domain, the spectral content representing the fringe pattern is effectively bandlimited by the speckle pattern given the conditions of Fig. 4 . Figure 5 shows the power spectra corresponding to the intensity distribution.

Investigating the features of Figs. 5-7 reveals the characteristic low-frequency speckle band that isotropically occupies the spatial frequency spectrum around the origin along with the sharp peak that originates from the image of the illumination spot. Additionally, the presence of two high-frequency sidebands located on the $\xi_{x}$ axis is observed in Figs. $\underline{5}$ and

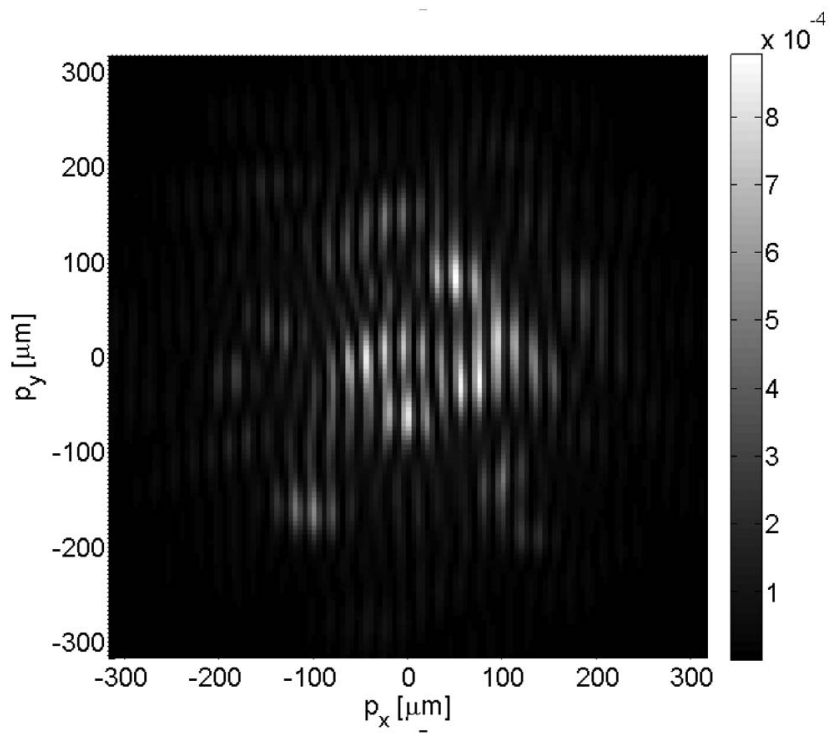

Fig. 4. Intensity distribution for $w=250 \mu \mathrm{m}, f=250 \mathrm{~mm}$, $P_{o}=P_{r}, \theta_{x}=0.0316 \mathrm{rad}, \theta_{y}=0 \mathrm{rad}$, and $\sigma=1.5 \mathrm{~mm}$.
6 . These sidebands originate from the regular fringe pattern. The above-mentioned band-limiting effect of the fringe pattern can be observed in Fig. 6 . The effect is evident when the aperture in the Fourier plane of the imaging system is changed, and thus the average speckle size is changed as well. As a result, the width of the spatial fringe pattern band changes according to the width of the spatial speckle band.

Based on observations from Fig. 5, the spatial periods and spatial filter directionality can be determined to optimize the subsequent signals that form the basis of the velocity measurements. Table 1 summarizes these selections, and Fig. 8 shows the power spectrum of the intensity distribution along with the spectral peak locations of the passbands for the spatial filters.

In order to calculate the velocity components $v_{x}, v_{y}$, and $v_{z}$ of the simulated particle distribution in the object plane, the time-dependent instantaneous output signal $i(t)$ of the spatial filters is considered. This quantity can be obtained from

$$
i(t)=\int_{-\infty}^{\infty} \mathrm{d}^{2} \mathbf{p} I(\mathbf{p}, t) h(\mathbf{p}) .
$$

In the case of a discrete intensity distribution, the integral of Eq. (25) is replaced by the corresponding summation operation. The measurement of a given velocity component is obtained by observing the temporal power spectrum of Eq. (25) given the response function $h(\mathbf{p})$ of the spatial filter addressing its respective velocity component. The particles in the object plane translate in the plane with the following velocities $v_{x}=\Delta x / \tau, v_{y}=\Delta y / \tau$, where $\tau$ is the time between samples. This means that each time the output signal is sampled, the particle field is translated one spatial period (or pixel) in both the $x$ and $y$ directions. The corresponding normalized frequencies, which are expected to be observed in the temporal power spectra of the output signals, can be

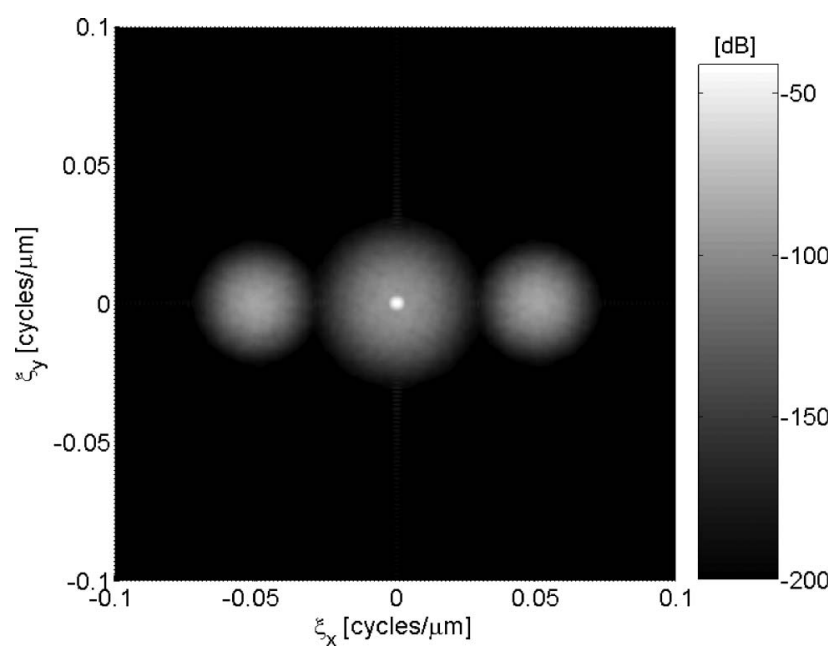

Fig. 5. Ensemble averaged spatial power spectrum $\left|S_{I}\left(\xi_{x}, \xi_{y}\right)\right|^{2}$ for $\theta_{x}=0.0316 \mathrm{rad}$, and $\theta_{y}=0 \mathrm{rad}$. The data set is the result of averaging 50 power spectra. 


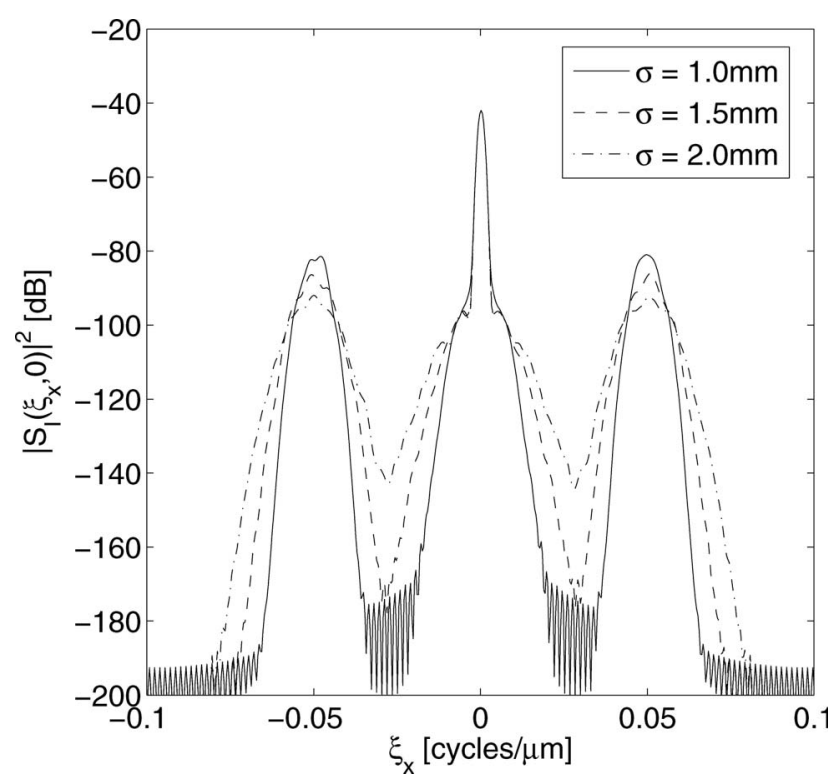

Fig. 6. Cross section along $\xi_{x}$ axis of the spatial power spectrum in Fig. 5 for different values of the aperture size $\sigma$.

obtained from Eq. (16), are given by

$$
f_{x}^{\mathrm{FUN}}=F_{x} \tau=\frac{\Delta x}{\Lambda_{x}}=\frac{5 \mu \mathrm{m}}{80 \mu \mathrm{m}}=\frac{1}{16},
$$

where $F_{x}$ is given by Eq. (16) for $v=v_{x}$. The equivalent expression is valid for $f_{y}^{\mathrm{FUN}}$. The out-of-plane velocity is selected so that $v_{z}=\lambda /(8 \tau)$. The fringe pattern will undergo a full oscillation in response to an out-of-plane displacement of $\lambda / 2$. Because the spatial frequency of the fringe pattern is arranged so that a spatial period exactly matches the spatial period of the spatial filter, a conversion factor of the actual axial displacement to the in-plane translation of the fringe pattern, must be taken into account. This factor can be written as $\alpha=2 \Lambda_{z} / \lambda$. Therefore, the normalized frequency is expected to be

$$
f_{z}^{\mathrm{FUN}}=F_{z} \tau=\frac{\alpha v_{z}}{\Lambda_{z}} \tau=\frac{1}{4} .
$$

In Fig. 9 the temporal power spectra of the output signals are shown along with the expected frequencies as given by Eq. (26) and (27) displayed as vertical lines.

Figure 9 shows that the spectral position of all three velocity components support the theoretical predic-

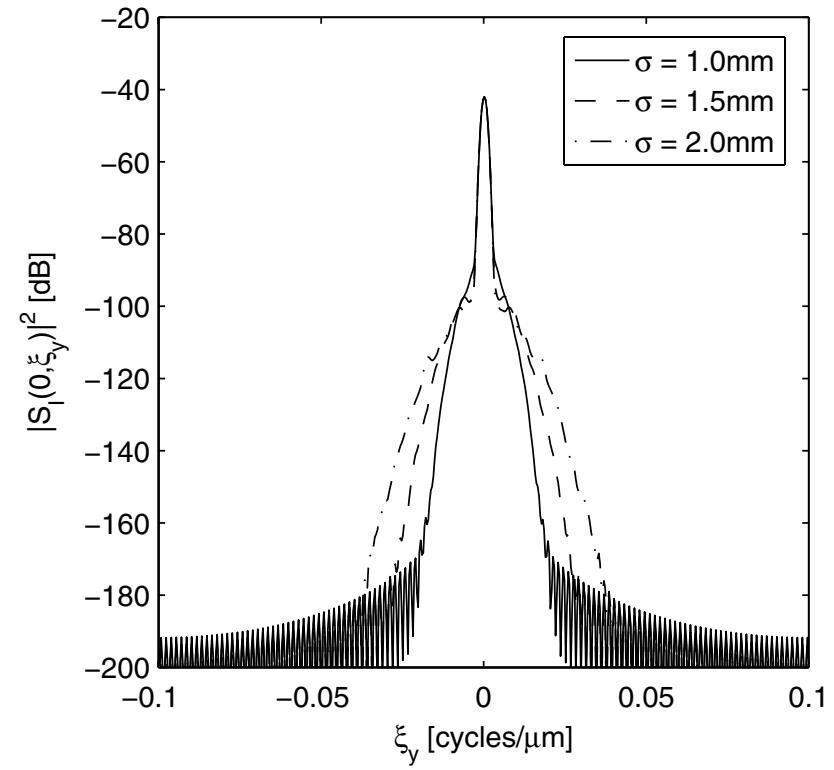

Fig. 7. Cross section along $\xi_{y}$ axis of the spatial power spectrum in Fig. $\underline{5}$ for different values of the aperture size $\sigma$.

tions of Eqs. (26) and (27). However, two additional features denoted $f_{x z}^{(-)}$and $f_{x z}^{(+)}$appear in the temporal spectrum of the signal from the spatial filter, measuring the $v_{x}$ component. The spectral peaks at the frequency positions denoted $f_{x z}^{(-)}$and $f_{x z}^{(+)}$originate because the third and fifth harmonic passbands of the spatial filter, measuring the $v_{x}$ component, overlap with the spatial frequency band occupied by the fringe pattern - as can be observed from Fig. 8. A more detailed study of the spectral positions of these two peaks confirms the following dependencies:

$$
\begin{aligned}
& f_{x z}^{(-)}=f_{z}^{\mathrm{FUN}}-f_{x}^{\mathrm{FUN}}, \\
& f_{x z}^{(+)}=f_{z}^{\mathrm{FUN}}+f_{x}^{\mathrm{FUN}} .
\end{aligned}
$$

These "crosstalk" frequencies can disrupt the velocity determination, especially due to their dynamic behavior. However, a solution to this problem can be identified: if the angle of incidence of the reference wave is changed so that

$$
\boldsymbol{\theta}=\left(\lambda /\left(\Lambda_{z} \sqrt{2}\right), \lambda /\left(\Lambda_{z} \sqrt{2}\right)\right),
$$

then the spatial period of the fringe pattern is numerically preserved, but the orientation is changed

Table 1. Spatial Passband Center Frequencies for the Spatial Filters Used in the Numerical Model

\begin{tabular}{cccc}
\hline & \multicolumn{2}{c}{ Velocity Component } \\
\cline { 2 - 3 } & \multicolumn{1}{c}{ Center Frequency $($ Cycles $/ \mu \mathrm{m})$} \\
\cline { 2 - 4 } First Order and Its Harmonics & $\Lambda_{x}=80 \mu \mathrm{m}, \Omega=0^{\circ}$ & $v_{y}$ & $\Lambda_{z}=80 \mu \mathrm{m}, \Omega=90^{\circ}$ \\
\hline First & $\pm 1 / 80$ & $\pm 1 / 80$ & $\Lambda_{z}=20 \mu \mathrm{m}, \Omega=0^{\circ}$ \\
Third & $\pm 3 / 80$ & $\pm 3 / 80$ & $\pm 1 / 20$ \\
Fifth & $\pm 5 / 80$ & $\pm 5 / 80$ & $\pm 3 / 20$ \\
\hline
\end{tabular}




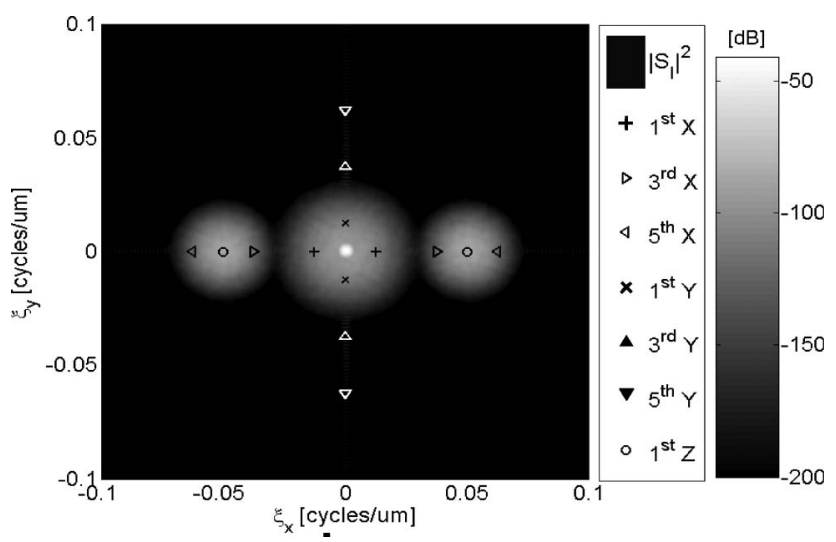

Fig. 8. Ensemble averaged spatial power spectrum $\left|S_{I}\left(\xi_{x}, \xi_{y}\right)\right|^{2}$ for $\theta_{x}=0.0316 \mathrm{rad}, \theta_{y}=0 \mathrm{rad}$ shown along with the center frequency locations of spatial frequency bands corresponding to spatial filters observing the $v_{x}, v_{y}$, and $v_{z}$ components of translation. The data set is the result of averaging 50 power spectra.

by $45^{\circ}$. If the spatial filter observing $v_{z}$ component is implemented with $\Omega=45^{\circ}$, then the measurement of $v_{z}$ is maintained while the spatial frequency band of the fringe pattern is isolated from the passbands of the spatial filter measuring the $v_{x}$ component. This configuration was implemented in the experimental setup and a discussion of the effects is included in Section $\underline{6}$.

\section{Experiment}

The experimental data are obtained using the setup illustrated in Fig. 10.

The coherent light source used in this experiment is a polarization-controlled $\mathrm{He}-\mathrm{Ne}$ laser operating at a wavelength of $\lambda=633 \mathrm{~nm}$. The radiation from the laser is collimated to a spot size of $\sim 1 \mathrm{~mm}\left(1 / e^{2}\right.$ intensity radius) with a beam expander. The expanded beam is split by a nonpolarizing beam splitter (BS). One of the beams is directed toward the object and forms the illumination beam. The other beam is directed toward the observation plane coinciding with the image of the object under an angle of incidence of $\boldsymbol{\theta}=(0.030 \mathrm{rad}, 0.030 \mathrm{rad})$ relative to the optical axis of the imaging system, thereby establishing the reference wave. The object is mounted on a translation stage, and it consists of an aluminum surface that has been treated to ensure a suitable rough surface

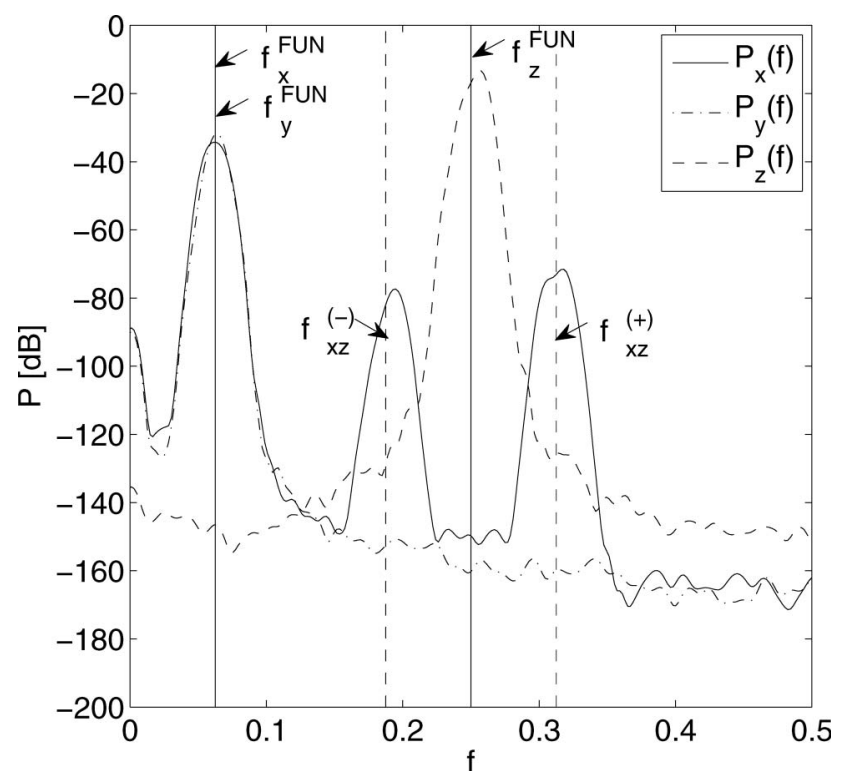

Fig. 9. Temporal power spectra as a function of normalized frequency obtained by FFT. The quantity $P_{i}(f)$ for $i=\{x, y, z\}$ denotes the power spectrum of the output signal of the spatial filter observing the $v_{x}, v_{y}$, and $v_{z}$ components, respectively. The data set is the result of averaging 10 power spectra.

for generation of a fully developed speckle pattern. The direction of translation can be adjusted to ensure both in-plane and out-of-plane translation with respect to the measurement directions of the spatial filters. Finally, the speed of translation can be varied.

The object plane is imaged onto the observation plane with a clean imaging system [11] consisting of two lenses and an adjustable diameter aperture placed in the Fourier plane of the system. The focal lengths of the two lenses are $f_{1}=f_{2}=250 \mathrm{~mm}$. This configuration is identical to the optical system emulated with the ABCD matrices described in Section 3, apart from the aperture. In the experimental setup, the amplitude transmittance function is circular with a radius of $\sigma=1 \mathrm{~mm}$ rather than apodized with a Gaussian function. For this specific imaging system, the depth of focus $\left(d_{o}\right)$ is approximately $\sim 25 \mathrm{~mm}$.

By implementing a 50:50 nonpolarizing BS immediately behind the second lens $\left(f_{2}\right)$ of the imaging system, the observation plane is effectively split into two. This enables implementation of two separated

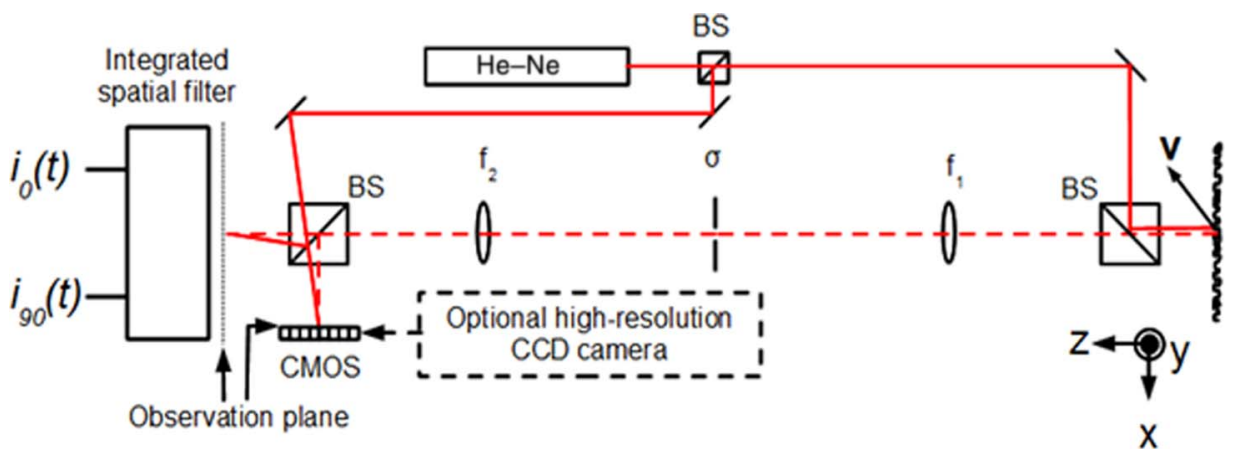

Fig. 10. (Color online) Experimental setup. 
systems for velocity measurement. In order to measure the in-plane velocity components, a CMOS detector array is utilized. The CMOS array used here consists of a $256 \times 256$ matrix of detectors with pixel dimensions of $10.6 \mu \mathrm{m} \times 10.6 \mu \mathrm{m}$. A frame-grabber records a sequence of images as the object translates. The spatial filtering operation is implemented by means of the method described in Section 3.

The out-of-plane velocity measurement is performed by an integrated optical spatial filter that is rotated corresponding to $\Omega=45^{\circ}$ in relation to the observation plane coordinate system, whereas the CMOS detector array is orientated in a configuration that is equivalent to $\Omega=0^{\circ}$. Figure 11 illustrates the integrated optical spatial filter.

The optical spatial filter in this device is injection molded and combined with a detector array containing four elements. The optical spatial filter consists of an array of cylindrical lenses (lenslets) separated by a distance of $\Lambda_{z}$. The radius of curvature of the lenslets is denoted $R_{3}$. Each of the lenslets in the array forms a one-dimensional imaging system with a large, single lens on the back side of the optical element. This lens has a radius of curvature of $R_{4}$. The photocurrents generated by the four individual detectors in the array are converted into voltage signals and directed to a set of differential amplifiers in the manner illustrated in Fig. 11. The detectors are arranged so that

$$
d_{180}=\frac{\Lambda_{z} R_{4}}{2 R_{3}}
$$

Given the relationship of Eq. (31), a backpropagation of the detector array through the optical system [16] will result in a reduced and repeated image of the detector array being projected to the input plane of the spatial filter device. If the lenslet array contains $N$ lenslets, and assuming perfect imaging, the spatial transfer function of this system will be given by Eq. (21) where the width of the filter in its direction of periodicity is $L_{z}=N \Lambda_{z}$ and the width of a single backpropagated detector is $W_{d}=\Lambda_{z} / 4$. The output signals from the two differential amplifiers are digitized by an analog-to-digital converter, resulting in an in-phase signal and a signal in phase quadrature for directional discrimination. A more detailed description of this type of system can also be found in [14].

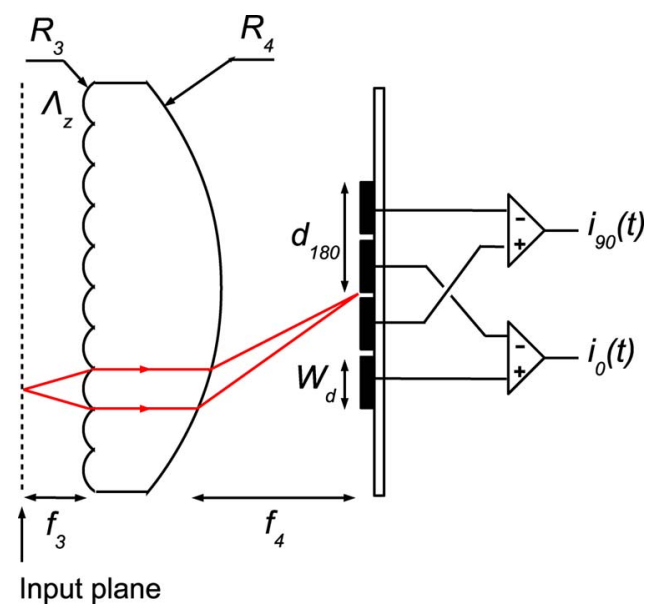

Fig. 11. (Color online) Integrated optical spatial filter. The physical parameters of the filter are $\Lambda_{z}=15 \mu \mathrm{m}, R_{3}=18 \mu \mathrm{m}$, $R_{4}=1 \mathrm{~mm}$, and $W_{d}=187.5 \mu \mathrm{m}$.

Table 2 lists the parameters and orientation of the spatial filters used in the measurements.

\section{Results}

Using a high-resolution camera, an intensity distribution in the observation plane was recorded. This distribution is shown in Fig. 12. This shows that the fringe pattern is enveloped by the speckle patterns as expected. The fringe pattern is oriented so that the fringes are parallel with the cylindrical lenslets of the integrated optical spatial filter, and the spatial frequency of the fringes matches the fundamental passband of the filter.

In Fig. 13, the spatial power spectrum of the intensity distribution is shown along with the center frequencies of the passbands of the spatial filters. It can be observed that the frequency distributions of both the fringe and speckle signal segments exhibit a different functionality than the frequency distributions obtained in the numerical simulations. This is due to the transmission function of the aperture in the Fourier plane of the imaging system being circular [15] in the experimental configuration rather than Gaussian as was the case in the numerical simulations.

Comparing the signals in the spatial frequency domain with the center frequencies of the passbands listed in Table 2, it can be observed that the fundamental frequencies of all three filters for in-plane and out-of-plane velocity measurements overlap their respective signal segments.

Table 2. Spatial Passband Center Frequencies for the Spatial Filters Used in the Experiment

\begin{tabular}{cccc}
\hline & \multicolumn{2}{c}{ Velocity Component } \\
\cline { 2 - 3 } & \multicolumn{3}{c}{ Center Frequency $($ Cycles $/ \mu \mathrm{m})$} \\
\cline { 2 - 4 } First Order and Its Harmonics & $v_{x}=84.8 \mu \mathrm{m}, \Omega=90^{\circ}$ & $\Lambda_{y}=84.8 \mu \mathrm{m}, \Omega=0^{\circ}$ & $\Lambda_{z}=15 \mu \mathrm{m}, \Omega=45^{\circ}$ \\
\hline First & \pm 0.0118 & \pm 0.0118 & \pm 0.0667 \\
Third & \pm 0.0354 & \pm 0.0354 & \pm 0.2000 \\
Fifth & \pm 0.0590 & \pm 0.0590 & \pm 0.3333 \\
\hline
\end{tabular}




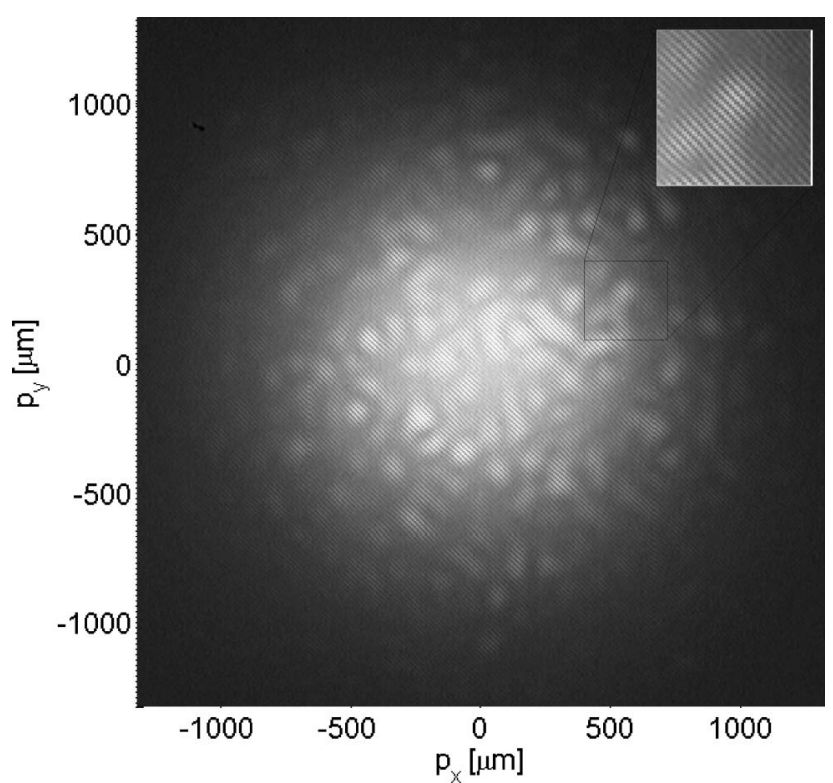

Fig. 12. Intensity distribution obtained using a high-resolution CCD camera.

The object is now arranged in such a way that the velocity components in relation to the measurement axis can be written as

$$
\begin{aligned}
\left(v_{x}, v_{y}, v_{z}\right)= & \left(v_{\mathrm{obj}} \cos \left(45^{\circ}\right) \sin \left(93.3^{\circ}\right), v_{\mathrm{obj}} \sin \left(45^{\circ}\right)\right. \\
& \left.\times \sin \left(93.3^{\circ}\right), v_{\mathrm{obj}} \cos \left(93.3^{\circ}\right)\right),
\end{aligned}
$$

where $v_{\text {obj }}$ is the (constant) speed of the object.

The time series of intensity distributions was recorded with the CMOS camera and processed with the numerically defined spatial filters, while the output signal of the optical spatial filter measuring the $v_{z}$ component was recorded using an oscilloscope. An object speed of $v_{\mathrm{obj}}= \pm 0.1 \mathrm{~mm} / \mathrm{s}$ was selected for the

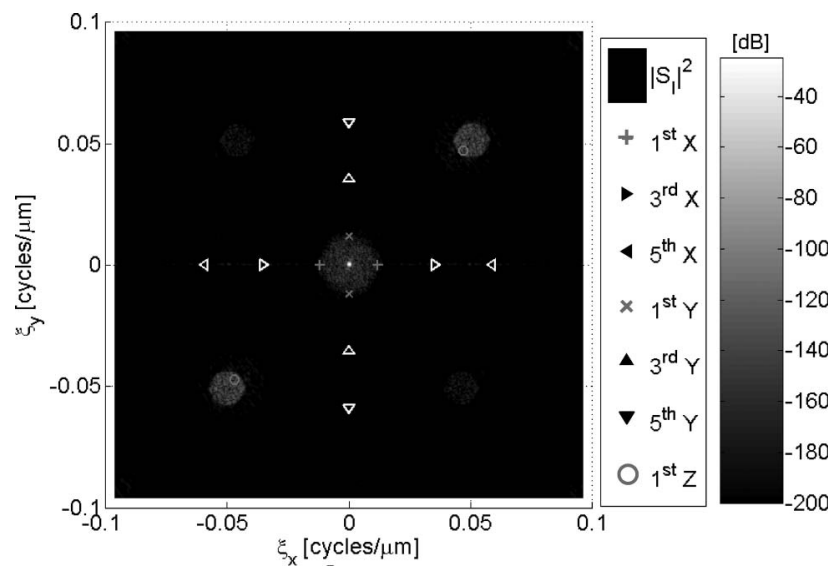

Fig. 13. Spatial power spectrum of the intensity distribution obtained with the high-resolution CCD camera.

top and bottom lines of the plots in Fig. 14. In the top of the individual plots, the entire signals are displayed. In the main plots, an excerpt corresponding to the interval outlined with the two vertical lines is shown. It can be observed that as the translation direction is reversed, the phase relationship between the in-phase and phase-quadrature signal changes sign, indicating a reversal of direction. This can be observed for all three measurements corresponding to the $v_{x}, v_{y}$, and $v_{z}$ components, demonstrating that the directionality of all three velocity components can be discriminated.

To measure the magnitude of the three velocity components, the temporal power spectrum of the spatial filter signals is considered for different values of $v_{\text {obj }}$.

Figures 15-17 show the power spectra of the spatial filter signals for observing the $v_{x}, v_{y}$, and $v_{z}$ components for four different values of $v_{\mathrm{obj}}$. The vertical

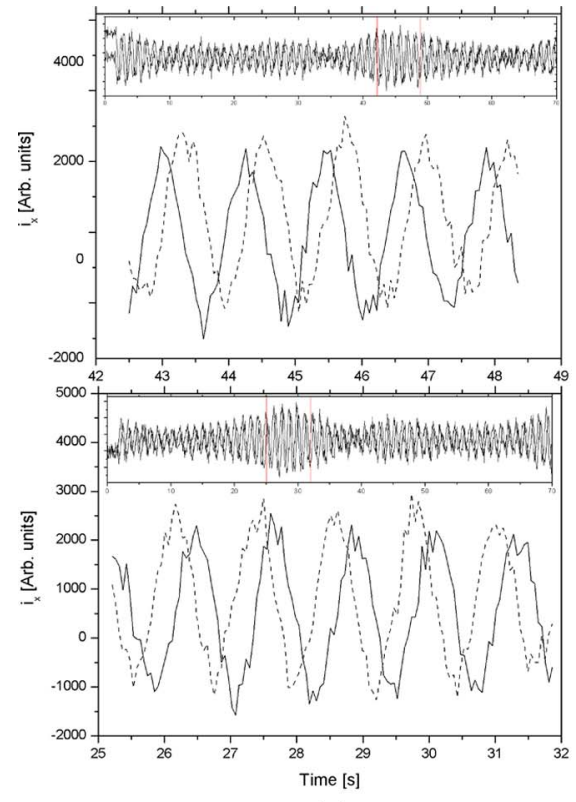

(a)

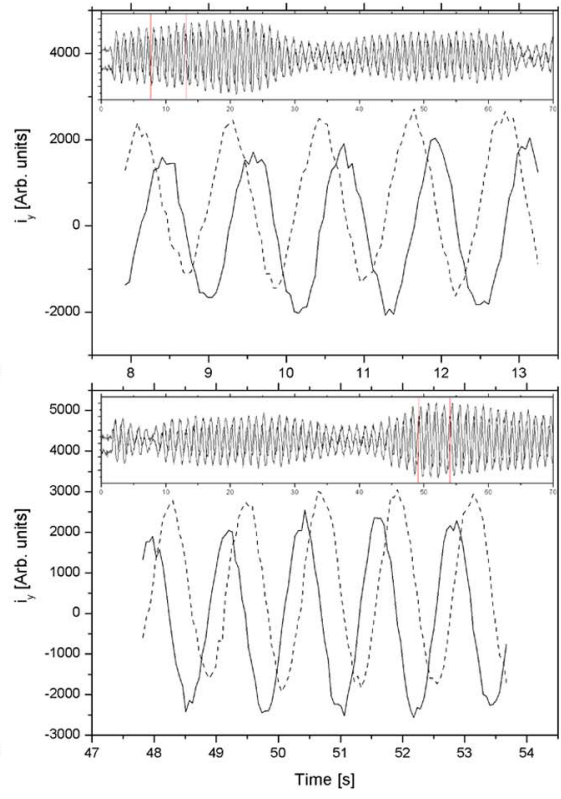

(b)

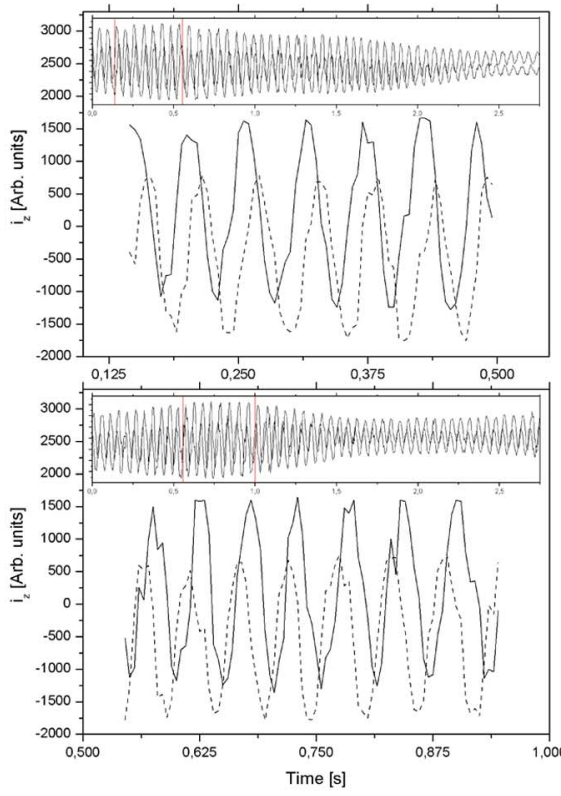

(c)

Fig. 14. (Color online) Spatial filter output signals. Vertical lines delimit the excerpts shown in the main plot. Top, $v_{\mathrm{obj}}=0.1 \mathrm{~mm} / \mathrm{s}$. Bottom, $v_{\mathrm{obj}}=-0.1 \mathrm{~mm} / \mathrm{s}$. Plots $(\mathrm{A})-(\mathrm{C})$ correspond to $v_{x}, v_{y}$ and $v_{z}$, respectively. 


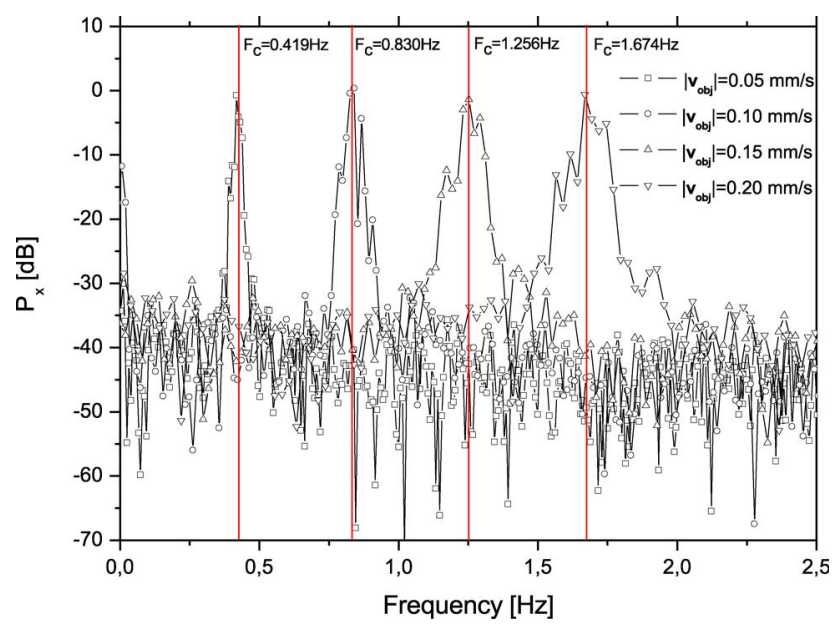

Fig. 15. (Color online) Power spectra of output signal from the spatial filter observing the $v_{x}$ velocity component for different values of $v_{\mathrm{obj}}$.

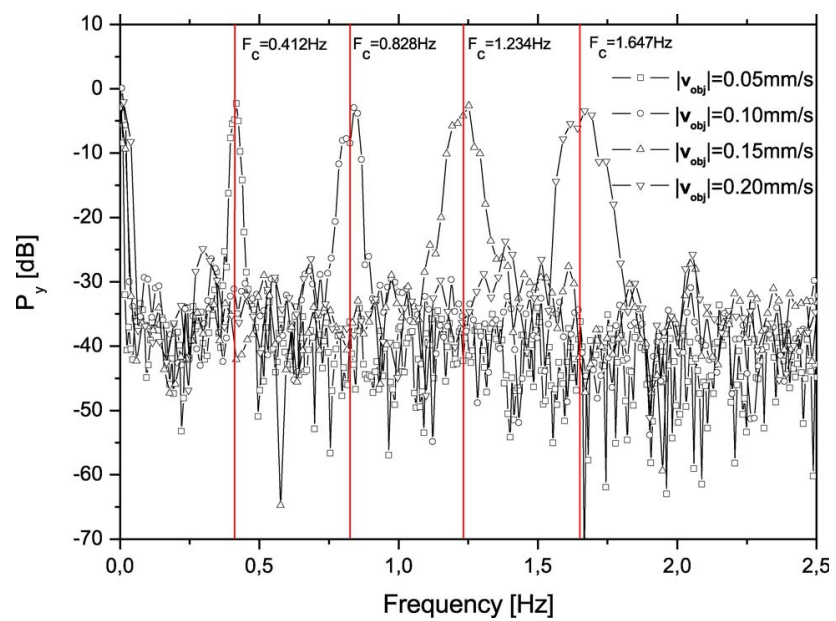

Fig. 16. (Color online) Power spectra of output signal from the spatial filter observing the $v_{y}$ velocity component for different values of $v_{\mathrm{obj}}$.

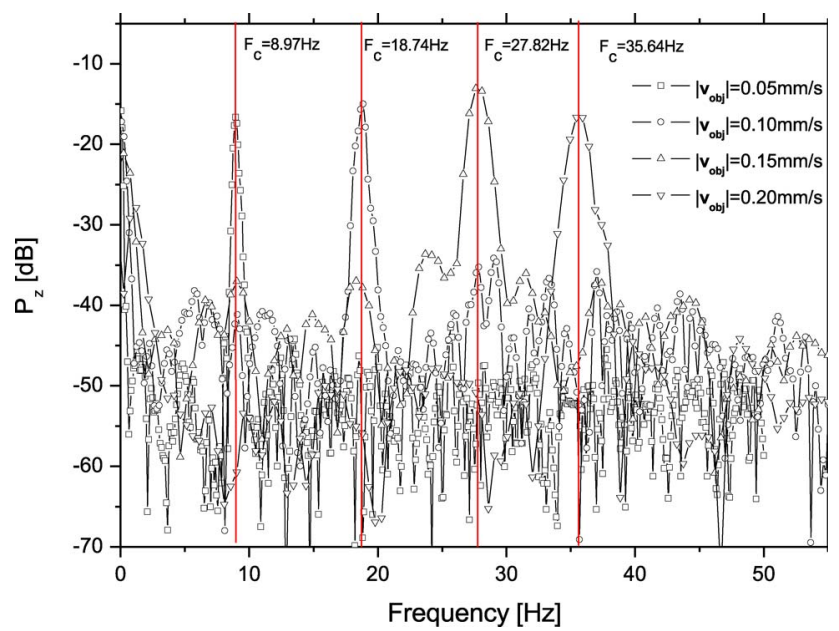

Fig. 17. (Color online) Power spectra of output signal from the spatial filter observing the $v_{z}$ velocity component for different values of $v_{\mathrm{obj}}$.
Table 3. Best Fitting Parameters of the Linear Relationship Relating the Measured Velocity to the Actual Velocity

\begin{tabular}{cccc}
\hline Velocity Component & $c_{0}(\mathrm{~mm} / \mathrm{s})$ & $c_{1}$ & $R^{2^{a}}$ \\
\hline$v_{x}$ & -0.0003 & 1.007 & 0.99996 \\
$v_{y}$ & 0.0001 & 0.987 & 0.99997 \\
$v_{z}$ & 0.0001 & 0.987 & 0.99658 \\
\hline
\end{tabular}

${ }^{a}$ Indicates the accuracy of the fit. When the value $R^{2} \rightarrow 1$, the model, in this case described by Eq. (33), approaches perfect correlation with the sample data, i.e., a perfect fit.

lines mark the measured center frequencies based on the centroid determined within a frequency window that is centered about the frequency peak and truncated at the noise floor. It is expected that there exists a linear relationship between the measured velocity components and the theoretically predicted values. This relationship is assumed to be of the form

$$
v_{i}^{\text {measured }}=c_{0}+c_{1} v_{i}^{\text {actual }}
$$

where $i=\{x, y, z\}$. The center frequencies obtained from the measurements are expected to be related to the velocity component through Eq. (16). In Table 3 , the best fitting values of $c_{0}$ and $c_{1}$ are listed for all three velocity components along with the associated correlation coefficient $\left(R^{2}\right)$.

\section{Discussion and Conclusion}

It has been demonstrated that all three velocity components of a diffusely scattering rigid object can be extracted using imaging speckles combined with an angular offset reference wave and methods based on spatial filtering velocimetry. This was achieved by tuning the fundamental spatial passbands of spatial filters to the speckle pattern and the regular fringe pattern, respectively. A numerical model was developed to determine the output of realistic spatial filter implementations such as a rectangular apodized detector arrangement. The numerical model showed that all three velocity components could, in principle, be extracted using a two-dimensional detector array. However, the model also predicted a crosstalk phenomenon that could impact the measurement negatively. A solution that solved this problem optically was identified. The fringe pattern orientation was changed by altering the angle of the incidence vector of the reference wave, effectively removing the spatial frequency component of the fringe pattern from the passbands of the higher harmonics of the spatial filters tuned to the speckle pattern.

Using a CMOS camera and an integrated optical spatial filter, the numerical model predictions were experimentally verified. It was successfully demonstrated that the direction of translation could be extracted for all three velocity components using a combination of an in-phase and phase-quadrature detector arrangement. The magnitude of each velocity component was varied by adjusting the speed of the translation stage. The measured velocities were in good agreement with the actual velocities. 
Generally, the accuracy with which the velocity can be determined using the proposed method depends on the specific experiment and implementation used. The limitation on measurement accuracy imposed by the spatial filters is related to the relative spectral width of the fundamental passband and is inversely proportional to the number of unit cells in the array constituting the filter. This number effectively determines how many signal oscillations can be observed. A treatment of the accuracy of spatial filtering velocimetry techniques can be found in [10]. The method described here relies on speckle translation to measure the transverse velocity components. The fringe pattern, used to measure the out-of-plane component, is limited by the correlation properties of the speckle pattern. Thus, speckle decorrelation (boiling) will be the limiting factor of the measurement accuracy of the transverse components if the speckle decorrelation length is significantly shorter than the spatial filter window $(L=N \Lambda)$. Similarly, when imaging the object, the depth of focus determines the axial correlation length of the speckles in the observation plane [17]. Thus, the average number of oscillations in the output signal of the spatial filter observing the out-of-plane component will be $\sim 2 d_{0} / \lambda$. Generally then, the system should be designed to accommodate the maximum number of unit cells in the spatial filter that will contribute constructively to the signal in order to maximize the number of signal oscillations. Fundamentally, the detection noise and finite measurement time will ultimately dictate the attainable accuracy.

The methodology described here is well suited for measuring translational velocity of rigid objects or surfaces. The velocities that were investigated were fairly small. This was due to a limited frame rate of the camera system. For high-speed applications, the use of integrated optical spatial filters to measure the in-plane velocity components, similar to that used for the measurement of the out-of-plane object translation, is required. Because of the fact that the detector array only contains four detectors and the spatial filtering is performed optically, these devices run routinely with sample rates in the megahertz regime.

Another interesting application is in flow velocity measurements-particles suspended in fluid or aerosols in the atmosphere. However, the effects of turbulence will increase the rate of decorrelation of the speckle pattern and thus reduce signal quality and availability. Also, the effects of noise will become critical as the backscattered signal becomes small, as is the case for probing atmospheric flows. These effects must be understood in detail to determine the viability of the methodology for such applications.

This work has been partly funded by OPDI Technologies A/S.

\section{References}

1. M. Sjödahl, "Some recent advances in electronic speckle photography," Opt. Lasers Eng. 29, 125-144 (1998).

2. P. Meinlschmidt, K. D. Hinsch, and R. S. Sirohi, eds., "Electronic Speckle Pattern Interferometry," Vol. MS 132 of SPIE Milestone Series (SPIE, 1996).

3. M. Sjödahl and H. O. Saldner, "Three-dimensional deformation field measurements with simultaneous TV holography and electronic speckle photography," Appl. Opt. 36, 36453648 (1997).

4. S. L. Yeh, S. T. Lin, and Y. H. Chang, "Precise displacement measurement for a local surface," Opt. Lett. 34, 3406-3408 (2009).

5. J. W. Bilbro, C. DiMarzio, D. Fitzjarrald, S. Johnson, and W. Jones, "Airborne Doppler lidar measurements," Appl. Opt. 25, 3952-3960 (1986).

6. R. S. Hansen and C. Pedersen, "All semiconductor laser Doppler anemometer at $1.55 \mu \mathrm{m}$," Opt. Express 16, 1828818295 (2008).

7. L. Z. Kennedy and J. W. Bilbro, "Remote intensity fluctuation measurements with a laser Doppler radar," Appl. Opt. 15, 2008-2008 (1976).

8. J. O'Shaughnessy and W. R. M. Pomeroy, "Single beam atmospheric transverse velocity measurement," Opt. Quantum Electron. 10, 270-272 (1978).

9. J. H. Churnside and H. T. Yura, "Laser vector velocimetry: a 3-D measurement technique," Appl. Opt. 21, 845-850 (1982).

10. Y. Aizu and T. Asakura, Spatial Filtering Velocimetry: Fundamentals and Applications (Springer-Verlag, 2006).

11. H. T. Yura and S. G. Hanson, "Optical beam wave propagation through complex optical systems,” J. Opt. Soc. Am. A 4, 19311948 (1987).

12. U. Schnell, J. Piot, and R. Dandliker, "Detection of movement with laser speckle patterns: statistical properties," J. Opt. Soc. Am. A 15, 207-216 (1998).

13. S. Bergeler and H. Krambeer, "Novel optical spatial filtering methods based on two-dimensional photodetector arrays," Meas. Sci. Technol. 15, 1309-1315 (2004).

14. M. L. Jakobsen and S. G. Hanson, "Lenticular array for spatial filtering velocimetry of laser speckles from solid surfaces," Appl. Opt. 43, 4643-4651 (2004).

15. J. W. Goodman, "Statistical properties of laser speckle patterns," in Laser Speckle and Related Phenomena, J. C. Dainty, ed. (Springer-Verlag, 1984), Chap. 2.

16. A. E. Siegman, "The antenna properties of optical heterodyne receivers," Appl. Opt. 5, 1588-1594 (1966).

17. H. T. Yura, S. G. Hanson, R. S. Hanson, and B. Rose, "Threedimensional speckle dynamics in paraxial optical systems," J. Opt. Soc. Am. A 16, 1402-1412 (1999). 